\section{BMJ Open Respiratory Research}

\title{
Randomised cross-over trial evaluating the short-term effects of non-invasive ventilation as an adjunct to airway clearance techniques in adults with cystic fibrosis
}

\author{
Gemma Stanford, ${ }^{\oplus 1}$ Helen Parrott, ${ }^{2}$ Diana Bilton, ${ }^{3}$ Penny Agent, ${ }^{2}$ Winston Banya, ${ }^{4}$ \\ Nicholas Simmonds ${ }^{1}$
}

\begin{abstract}
To cite: Stanford G, Parrott $\mathrm{H}$, Bilton D, et al. Randomised cross-over trial evaluating the short-term effects of non-invasive ventilation as an adjunct to airway clearance techniques in adults with cystic fibrosis. BMJ Open Resp Res 2019;6:e000399. doi:10.1136/ bmjresp-2018-000399
\end{abstract}

- Additional material is published online only. To view please visit the journal online (http://dx.doi.org/10.1136/ bmjresp-2018-000399).

Received 2 January 2019 Revised 5 March 2019 Accepted 20 March 2019

Check for updates

\section{(c) Author(s) (or their} employer(s)) 2019. Re-use permitted under CC BY-NC. No commercial re-use. See rights and permissions. Published by BMJ.

${ }^{1}$ Cystic Fibrosis, Royal Brompton Hospital, London, UK

${ }^{2}$ Rehabilitation and Therapies, Royal Brompton Hospital, London, UK

${ }^{3}$ Respiratory Medicine, Royal Brompton Hospital, London, UK

${ }^{4}$ Research and Development, Royal Brompton Hospital, London, UK

Correspondence to Gemma Stanford; g.stanford@rbht.nhs.uk

\section{ABSTRACT}

Introduction Non-invasive ventilation (NIV) is used in cystic fibrosis (CF) to support airway clearance techniques (ACTs) by augmenting tidal volumes and reducing patient effort. However, the evidence base for this is limited. We hypothesised that NIV, in addition to usual ACT, would increase sputum clearance. In addition, we investigated ease of sputum clearance $(\mathrm{E} O \mathrm{C})$, work of breathing (WoB) and NIV tolerability.

Methods Adults with CF (16+ years) at the end of hospitalisation for a pulmonary exacerbation were randomised to a cross-over trial of NIV-supported ACT or ACT alone in two consecutive days. No other changes to standard care were made. The primary outcome was the total 24-hour expectorated sputum wet weight after the intervention. Spirometry was completed pre-treatment and post-treatment. 0xygen saturations were measured pretreatment, during treatment and post-treatment. EoC and WoB were assessed using Visual Analogue Scale.

Results 14 subjects completed the study (7 male, mean age 35 [SD 17] years, mean forced expiratory volume in $1 \mathrm{~s}\left[\mathrm{FEV}_{1}\right] 49$ [20] \% predicted). The difference between treatment regimens was $-0.98 \mathrm{~g}$ sputum $(95 \% \mathrm{Cl}-11.5$ to $9.6, p=0.84$ ) over 24 hours. During treatment oxygen saturations were significantly higher with NIV-supported ACT (mean difference 2.0, 95\% $\mathrm{Cl} 0.9$ to 2.6, $\mathrm{p}=0.0004$ ). No other significant differences were found in posttreatment $\mathrm{FEV}_{1}$, EoC, WoB, oxygen saturations or subject preference.

Conclusions There was no difference in treatment effect between NIV-supported ACT and ACT alone, although the study was underpowered. Oxygen saturations were significantly higher during NIV-supported ACT, but with no effect on post-treatment saturations. NIV was well tolerated.

Trial registration number NCT01885650.

\section{BACKGROUND}

Cystic fibrosis (CF) is a genetic disorder affecting over 10000 people in the UK. ${ }^{1} \mathrm{CF}$ is characterised by the production of thickened bodily secretions, affecting the function
Key messages

Does the addition of positive pressure support via the use of non-invasive ventilation (NIV) during chest physiotherapy improve sputum clearance for adults with cystic fibrosis (CF) compared with their usual airway clearance technique (ACT)?

- This research concludes that while NIV has not been shown to be superior to the usual ACT, we cannot rule out that it has no clinical benefit especially for patients with lower lung function or who are acutely unwell, and so it is an important tool to be considered when assessing a person's ACT needs.

- This work discusses the outcomes of a randomised controlled cross-over trial investigating the use of NIV with ACT in adults with CF who were inpatients at our CF unit, and the difficulties of conducting research in this patient group, alongside the issues that investigators of ACT face due to the lack of accepted robust outcome measures available at present time.

of organs such as the pancreas and the lungs due to altered ion transport across cell membranes. Within the lungs, dehydration of the airway surface liquid alters normal mucociliary clearance mechanisms, resulting in airway obstruction, mucus plugging and infections. ${ }^{2}$ Recurrent infections and subsequent inflammation are thought to be the major mechanism towards lung tissue damage and the occurrence of fibrosis, ${ }^{34}$ which decreases lung function, lowers tissue oxygenation, and eventually leads to respiratory failure and death. Traditional CF management includes prevention of these complications by optimising removal of airway secretions. Combinations of mucolytic or hydrator therapy to make the secretions less viscous, and chest physiotherapy to mobilise secretions and 
improve airway clearance, are prescribed on an individual basis. $^{5}$

There are a range of airway clearance techniques (ACTs) available for patients with CF, including breathing methods such as the active cycle of breathing techniques (ACBT) or autogenic drainage (AD), adjuncts such as positive expiratory pressure (PEP), and oscillatory devices such as the flutter or acapella. Selection of the optimal ACT is driven mostly by patient preference and thorough assessment and teaching by a trained physiotherapist, ${ }^{6}$ as current research indicates no differences in the effectiveness of techniques ${ }^{7}$ as long as they are performed correctly and regularly. During a pulmonary exacerbation or in end-stage severe disease, breathlessness, fatigue or excessive secretions can inhibit effective airway clearance. The addition of positive pressure via non-invasive ventilation (NIV) to ACTs has been shown to decrease patient fatigue $\mathrm{e}^{8-11}$ and respiratory rate during clearance. ${ }^{8-10}$ Improvements in oxygenation, ${ }^{8}$ respiratory muscle strength, ${ }^{8}$ lung clearance index (LCI) ${ }^{12}$ and forced expiratory volume in 1 second $\left(\mathrm{FEV}_{1}\right)^{11}$ have also been reported.

To date, no research has reported an increase in sputum expectorated with the addition of positive pressure; however, the ability to augment greater tidal volumes through positive pressure is thought to be a mechanism which could improve sputum clearance. We have previously reported the subjective viewpoints of patients using NIV to support ACT, in which they felt their sputum clearance had increased. ${ }^{13}$ The aim of the present study was to investigate this observation objectively by assessing sputum cleared in 24 hours in a randomised cross-over trial of usual ACT versus NIV-supported ACT.

We hypothesised that the inclusion of NIV in a patient's normal ACT would objectively increase sputum clearance. In addition, we hypothesised that NIV would improve ease of sputum clearance (EoC) and work of breathing (WoB) and would be well tolerated.

\section{METHODS}

\section{Patient and public involvement}

The research question stemmed from previously published research interviews completed with $\mathrm{CF}$ patient users of NIV for ACT, ${ }^{13}$ which on further discussion with these patients the authors felt was important to try and answer objectively. Patients with CF from the Royal Brompton Hospital were involved in designing the methodology to try and answer the question of whether sputum clearance changed with the addition of NIV. This same patient group made up the participants of this study, who were able to assess the burden of intervention themselves with the information provided prior to them giving written and informed consent. The results of the study were disseminated to participants via a letter.

\section{Recruitment}

Subjects were recruited from the adult CF centre at Royal Brompton Hospital, London. They were eligible to participate in the trial if they had a diagnosis of CF confirmed by standard criteria, ${ }^{14}$ were $\geq 16$ years old, had been hospitalised for a pulmonary exacerbation which was resolving (by a clinician's assessment), had been an inpatient for $\geq 7$ days and had an established ACT regimen. Exclusion criteria included haemoptysis, pneumothorax, dependency on positive pressure support for ACT, history of spontaneous rib fracture, pregnancy, inability to give consent for participation, inclusion in another research study and requiring more than two supervised ACT sessions a day. Discontinuation criteria included voluntary withdrawal from the study, discharge from hospital prior to completion of the study or evidence of clinical deterioration during the study that required a change in medical treatment as determined by the CF medical team.

Written informed consent was gained prior to randomisation. Subjects were recruited between July 2013 and May 2017.

\section{Treatment}

The trial was a randomised cross-over design over 2 days, with subjects completing 1 day of their usual ACT regimen and 1 day of NIV-supported ACT using the Breas iSleep 25 (Breas Medical, Sweden). Randomisation was completed via a computerised program created by the trial statistician. Subjects were familiarised with NIV-supported ACT prior to randomisation. NIV settings were individually determined by a specialist physiotherapist based on the patient's comfort and augmented thoracic expansion for each subject. NIV was administered via a mouthpiece with a Portex Thermovent heat and moisture exchange filter (Smiths Medical, UK) within the circuit. During the NIV session subjects completed 10 supported NIV breaths followed by up to four repetitions of the forced expiration technique and coughing as required. During the usual ACT subjects completed similar routines of forced expirations and coughs. Subjects received two 30 min sessions of supervised ACT a day from an adult $\mathrm{CF}$ specialist physiotherapist trained in NIV (GS). The same physiotherapist completed $93 \%$ of all trial treatment sessions and NIV familiarisation. No other changes to usual clinical care occurred during the trial period. No adverse events occurred during either treatment arm during the trial period.

\section{Outcome measures}

The primary outcome measure was the 24-hour total sputum wet weight expectorated timed from initiation of the first ACT session of a research day to the same time the following day. The secondary outcome measures were qualitative assessment of $\mathrm{EoC}$, WoB during clearance and treatment satisfaction using a $10 \mathrm{~cm}$ Visual Analogue Scale (see online supplementary material 1), 
sputum expectorated during ACT sessions, $\mathrm{FEV}_{1}$, forced vital capacity (FVC), maximum expiratory flow at $25 \%$ of FVC (MEF25), maximum expiratory flow at $75 \%$ of FVC (MEF75), and oxygen saturations during ACT and post-ACT. Blinded independent observers completed all the outcome measurements pretreatment, immediately post-treatment and $30 \mathrm{~min}$ post-treatment, and weighed the 24-hour sputum. Oxygen saturations were recorded during the session and downloaded afterwards by the research team. At the end of the study, subjects completed a questionnaire asking their preferences on the different treatment regimens (see online supplementary material 2 ). Spirometry was completed according to the American Thoracic Society/European Respiratory Society guidelines ${ }^{15}$ using the MicroLoop hand-held spirometer (CareFusion UK 306, Basingstoke, UK; supplier: Williams Medical Supplies, Rhymney, UK). Oxygen saturations were recorded using a Pulsox-3i oximeter (Konica Minolta, Japan; supplier: Stowood Scientific Instruments, Stowood, UK).

\section{Statistical analysis}

A sample size calculation was completed (powered at $80 \%$ with $5 \%$ significance level) based on assuming an $\mathrm{SD}$ of $7.3 \mathrm{~g}$ for expectorated sputum, which required 28 participants to detect an absolute difference of 4 $\mathrm{g}$ of sputum. The sample size was calculated using the results from a previous randomised controlled trial on treatment effects. ${ }^{16}$ Data were described using mean (SD) or median (range) if non-parametric. Pretreatment and post-treatment effects were reported using mean difference (MD) and 95\% CI, with paired t-tests completed to analyse significance levels. A $p$ value of $<0.05$ was considered significant. An unplanned interim analysis was approved for completion by the London Chelsea Research Ethics Committee and carried out at the halfway point of recruitment due to slow recruitment and to determine the feasibility of study completion.

The interim analysis showed that the difference between the two treatment arms was $-0.98 \mathrm{~g}$ sputum $(95 \% \mathrm{CI}-11.5$ to 9.6, $\mathrm{p}=0.84$ ) over 24 hours, with NIV-supported ACT clearing less than the usual ACT. Analysis by the trial statistician indicated that based on the true difference in the mean response of the matched pairs of the data collected, the trial would require over 6000 pairs of subjects to be able to reject the null hypothesis that the response difference was zero with probability (power) of 0.8 . There was a high (0.05) probability of a type I error associated with this test of the null hypothesis. Due to these results the decision was made to terminate the study early.

\section{RESULTS}

\section{Participant demographics}

Fourteen subjects completed the study, 7 were men, with a mean (SD) age of 35.3 (17.1) years and a mean $\mathrm{FEV}_{1}$ of 48.9 (20) \% predicted. Figure 1 illustrates the flow of recruitment, and table 1 shows the details of subjects' baseline characteristics.

\section{Treatment details}

The median NIV settings were an inspiratory positive airway pressure of $25 \mathrm{cmH}_{2} \mathrm{O}(\mathrm{IQR} 25-75,24.6-25)$ and an expiratory positive airway pressure of $5.8 \mathrm{cmH}_{2} \mathrm{O}(\mathrm{IQR}$ 25-75, 5-6).

A variety of usual ACTs were included, with $A D$ completed by five subjects $(36 \%)$, PEP by six subjects $(43 \%)$, an oscillatory device by two subjects $(14 \%)$ and ACBT by one subject (7\%).

\section{Before and after treatment results}

No significant differences in sputum (table 2), spirometry, or pre-treatment to post-treatment oxygen saturations were found (see online supplementary material 3).

\section{Comparisons between regimens}

The MD between treatment regimens indicated that NIV-supported ACT cleared $1 \mathrm{~g}$ less sputum over 24 hours $(95 \% \mathrm{CI}-11.5$ to 9.6$)$ than the usual ACT $(\mathrm{p}=0.84)$ (table 2). During treatment the mean oxygen saturations were significantly higher during NIV-supported ACT (95.7\%, SD 2.3) compared with the usual ACT $(94 \%$, SD 2.5) (MD 2.0, 95\% CI 0.9 to 2.6, $\mathrm{p}=0.004$ ) (figure 2). Post-treatment values for $\mathrm{FEV}_{1}(\mathrm{p}=0.16)$, FVC $(\mathrm{p}=0.97)$, MEF $25(\mathrm{p}=0.70)$ and MEF $75(\mathrm{p}=0.19)$ did not differ significantly between the two regimens (see online supplementary materials $1-3$ ). No significant differences in patient-reported outcome measures, which included EoC and WoB, were found (table 3).

The results of the questionnaire asking subjects' opinions of the two techniques were analysed for themes. Eight $(57 \%)$ subjects had a preference for the usual ACT and six (47\%) for NIV-supported ACT. The main reasons for preferring usual ACT were that the person was used to it $(n=4)$ and that the techniques were more portable $(n=3)$; the reasons for preferring NIV-supported ACT included feeling that it would be easier when feeling unwell $(n=4)$, feeling like they got deeper breath $(n=5)$ and that deeper deposits of sputum were cleared $(n=3)$.

\section{DISCUSSION}

Airway clearance, especially in severe CF, is a complex process, and physiotherapists and patients strive to find techniques which are effective, while easy to perform and low in effort. In this study we tested the hypothesis that the addition of NIV to the usual ACT would be more effective in clearing sputum than the usual ACT alone.

This study found no significant difference in the primary outcome measure (the amount of sputum cleared in 24 hours) between the usual ACT and NIV-supported ACT, which is comparable with the results of other studies investigating NIV and ACT, ${ }^{89}$ and agrees with findings of the recent Cochrane review on NIV for 


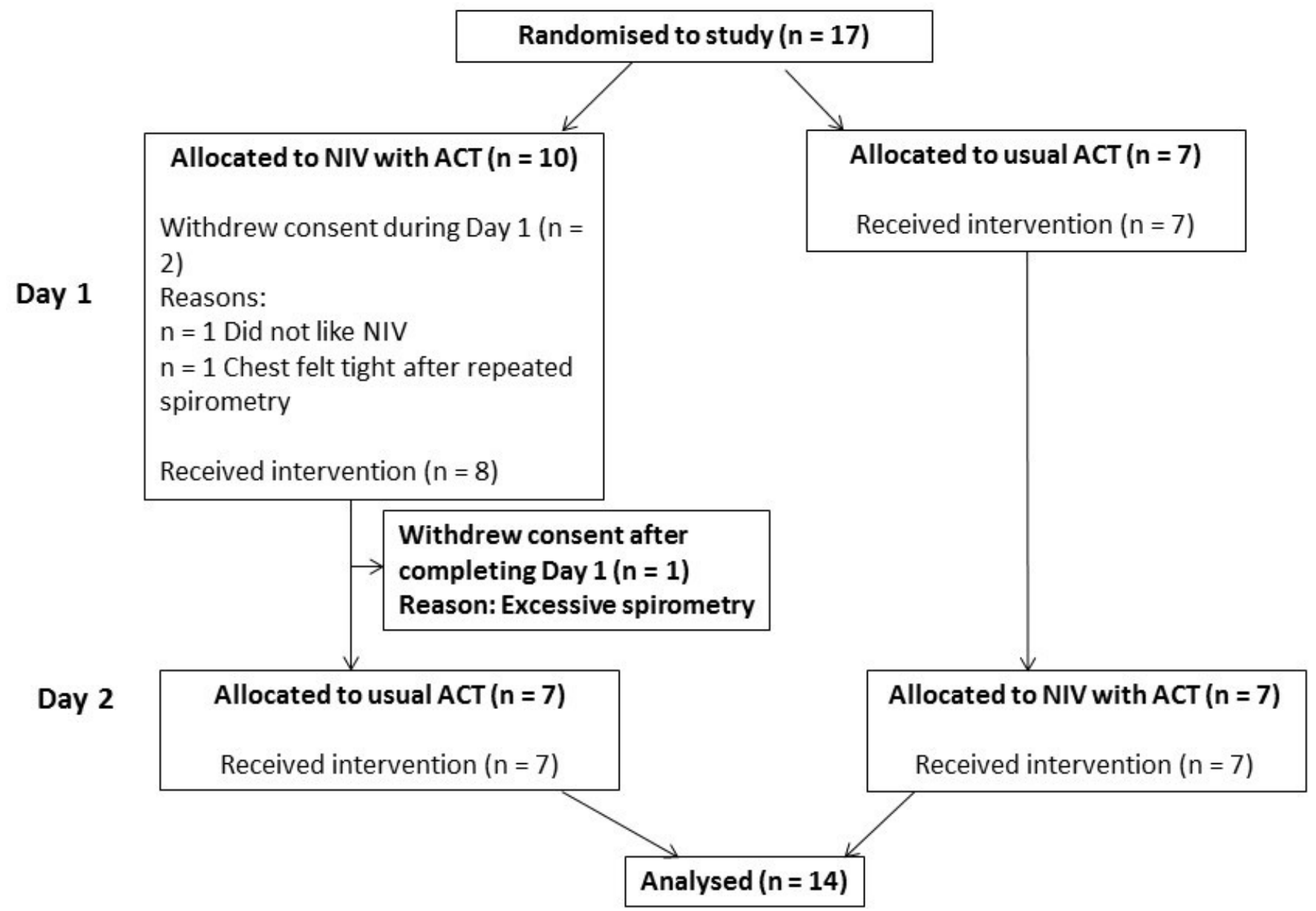

Figure 1 CONSORT diagram of the study. ACT, airway clearance technique; CONSORT, Consolidated Standards of Reporting Trials; 1 ; NIV, non-invasive ventilation.

\begin{tabular}{lc}
\hline Table $1 \quad$ Participant baseline characteristics & \\
\hline Baseline characteristics & $\mathbf{N}=14$ \\
\hline Mean age (SD), years & $35.5(17.1)$ \\
\hline Sex, female:male & $7: 7$ \\
\hline Mean FEV ${ }_{1}, \mathrm{~L}$ (SD) & $1.75(0.90)$ \\
Mean FEV ${ }_{1}, \%$ predicted (SD) & $48.9(20.1)$ \\
\hline Mean FVC, L (SD) & $2.88(1.15)$ \\
Mean FVC, \% predicted (SD) & $68.4(18.4)$ \\
Genotype, n (\%) & \\
Homozygous F508del & $7(50)$ \\
F508del + another mutation & $6(43)$ \\
2 other mutations & $1(7)$ \\
Main usual airway clearance technique, $\mathrm{n}(\%)$ & \\
Positive expiratory pressure & $6(43)$ \\
Autogenic drainage & $5(36)$ \\
Oscillatory device (flutter/acapella) & $2(14)$ \\
Active cycle of breathing techniques & $1(7)$ \\
Mucoactive agents, $\mathrm{n}(\%)^{*}$ & \\
\hline DNase & $13(93)$ \\
Hypertonic saline & $4(29)$ \\
Mannitol & $4(29)$ \\
\hline
\end{tabular}

*Some participants were taking more than one mucoactive agent. $\mathrm{FEV}_{1}$, forced expiratory volume in $1 \mathrm{~s}$; FVC, forced vital capacity.
CF. ${ }^{17}$ However, the study was significantly underpowered. Additional explanations for concluding no treatment difference include the short-term duration of the intervention and possibly due to NIV-supported ACT being comparable in effect with usual ACTs.

Significant differences were determined in oxygen saturations of the participants during the ACT session, with subjects having higher saturations when using NIV, although it can be argued that a $2 \%$ difference in oxygen level is not a clinically relevant difference. We also did not demonstrate a carryover of these improvements after the session, unlike Holland et al. ${ }^{8}$ During ACT sessions oxygen saturations are an important clinical consideration when completing ACT, especially with more acutely unwell patients or those with advanced lung disease, and it may be that this is an area where NIV-supported ACT has a role.

Airway clearance research has been debated within the physiotherapy, $\mathrm{CF}$ and wider research community as lacking in a robust outcome measure which appropriately measures the effect of ACT. Sputum weight has been criticised as being a subjective measure, based on the will of the subject to expectorate and at risk of contamination with saliva, which could alter the results. ${ }^{6}{ }^{18} \mathrm{FEV}_{1}$ is 
Table 2 Sputum pre-treatment, post-treatment and 30 min post-treatment

\begin{tabular}{lllll}
\hline & $\begin{array}{l}\text { Usual ACT } \\
\text { Mean (SD) }\end{array}$ & $\begin{array}{l}\text { NIV-supported ACT } \\
\text { Mean (SD) }\end{array}$ & MD (95\% Cl) & P value \\
\hline Sputum cleared after morning ACT session (g) & $12.0(5.0)$ & $13.0(6.18)$ & $1.0(-1.4$ to 3.3$)$ & 0.41 \\
Sputum total 30 min after morning ACT session (g) & $14.7(20.6)$ & $14.5(7.2)$ & $-0.2(-2.7$ to 2.3) & 0.86 \\
Sputum total after afternoon ACT session (g) & $35.0(20.6)$ & $30.6(15.2)$ & $-4.4(-12.2$ to 3.3$)$ & 0.24 \\
Sputum total 30 min after afternoon ACT session (g) & $36.7(21.4)$ & $32.6(15.5)$ & $-4.1(-12.1$ to 4.0$)$ & 0.29 \\
Total sputum cleared in 24 hours (g) & $49.0(29.4)$ & $48.1(30.8)$ & $-1.0(-11.5$ to 9.6$)$ & 0.84 \\
\hline
\end{tabular}

$\mathrm{ACT}$, airway clearance technique; MD, mean difference; NIV, non-invasive ventilation.

thought to be difficult to use as a short-term outcome due to its effort dependency and lack of sensitivity to small changes. ${ }^{671819}$ This may explain why we found no significant differences in $\mathrm{FEV}_{1}$ and other spirometric measures during this study. There was a slight trend for an increase in FVC after NIV-supported ACT, especially in the morning session, and although this did not reach statistical significance it may make NIV support a viable consideration in patients with loss of lung volume.

An alternative outcome measure which has been suggested is the LCI, which is derived from multiple breath washout. One recent study comparing NIV-supported ACT with PEP used the LCI as an outcome measure, and reported a significant reduction (improvement) in LCI when subjects used NIV compared with PEP. $^{12}$ Their study provides support for this outcome measure, although other published research investigating ACTs with LCI as an outcome have reported variable LCI results. ${ }^{20}$

A key aspect of ACT is the patient's own perspective, as fundamentally it is the patient who will be required to complete the technique daily. Using our self-designed questionnaires, subjects reported that NIV-supported ACT did not differ significantly from their usual ACT in terms of EoC, breathlessness, comfort or fatigue. There were trends for lower reported levels of breathlessness and fatigue with the use of NIV, but these did not reach significance. This differs from our previously reported results where subjects did subjectively rate NIV-supported ACT significantly higher for EoC and reduced effort ${ }^{13}$; however, those results were from a more severe patient group (median $\mathrm{FEV}_{1} 26 \%$ ) over a 1-year period. Several of the participants of the current study commented that they felt NIV would be useful for them if they were more unwell, breathless or unable to expectorate, as they felt it would be more supportive at that time, which may then provide more comparable data. This suggestion has been supported by Moran $\mathrm{et}_{\mathrm{al}}{ }^{17}$ in their recent Cochrane review and may be where NIV-supported ACT has a role. A consideration for future ACT studies is the timing of the intervention for maximal effect, as our subjects were nearing the end of admission, with many of them feeling

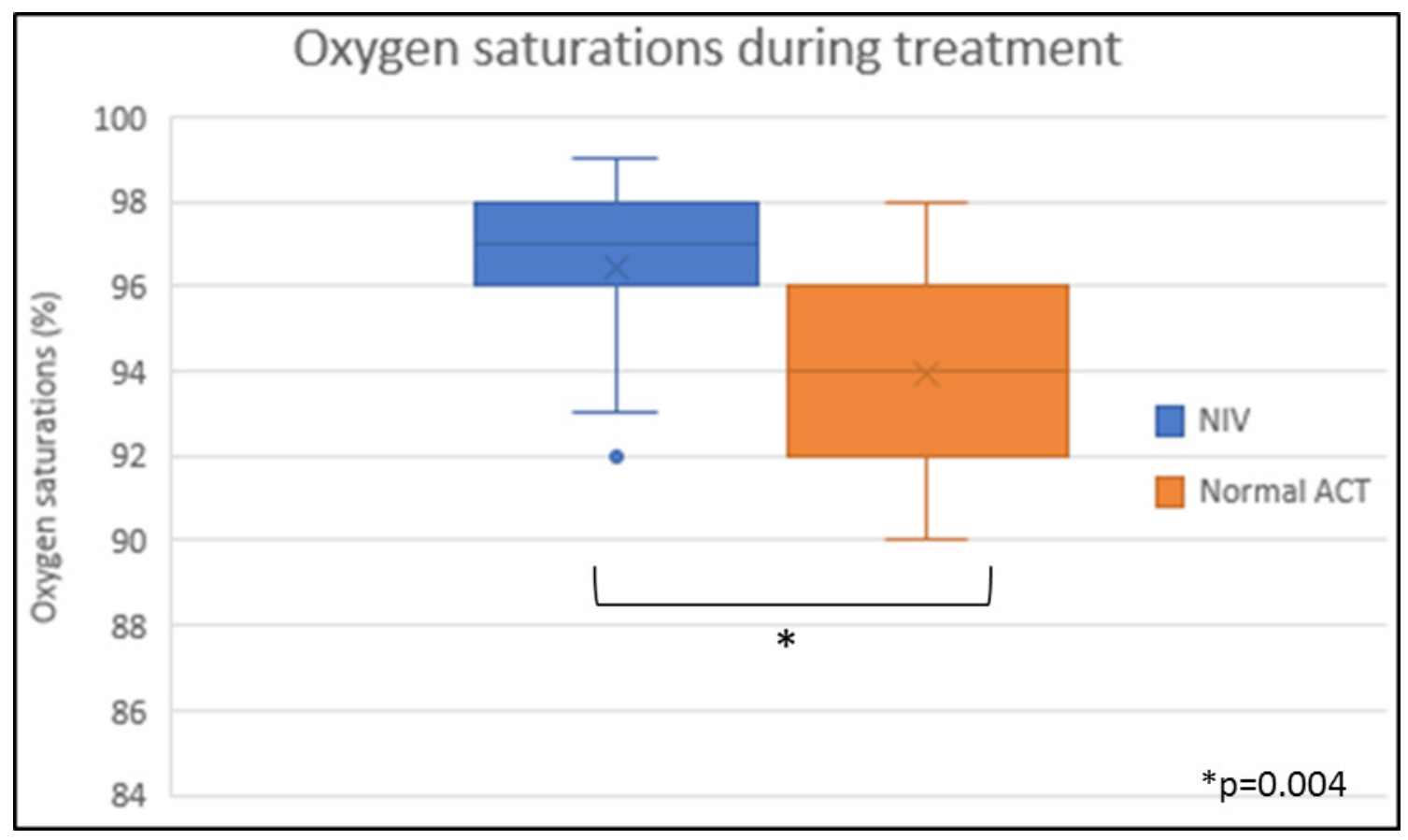

Figure 2 Oxygen saturation during treatment. ACT, airway clearance technique; NIV, non-invasive ventilation. 


\begin{tabular}{|c|c|c|c|c|}
\hline \multirow[b]{2}{*}{ Question } & \multicolumn{2}{|c|}{ Mean (SD) answers } & \multirow[b]{2}{*}{$\begin{array}{l}\text { Mean difference } \\
(95 \% \mathrm{Cl})\end{array}$} & \multirow[b]{2}{*}{$P$ value } \\
\hline & $\begin{array}{l}\text { NIV-supported } \\
\text { ACT }\end{array}$ & ACT alone & & \\
\hline $\begin{array}{l}\text { How breathless did you feel during your session using this } \\
\text { technique? } \\
\text { (0 least breathless, } 10 \text { most breathless) }\end{array}$ & $2.7(2.0)$ & $3.9(2.0)$ & $1.1(-2.4$ to 0.1$)$ & 0.08 \\
\hline $\begin{array}{l}\text { How tired did you feel during your physiotherapy session? } \\
\text { ( } 0 \text { least tired, } 10 \text { most tired) }\end{array}$ & $3.6(1.8)$ & $4.9(2.1)$ & 1.4 (-2.9 to 0.2$)$ & 0.07 \\
\hline $\begin{array}{l}\text { How comfortable did you find this technique? } \\
\text { ( } 0 \text { least comfortable, } 10 \text { most comfortable) }\end{array}$ & $6.1(2.2)$ & $6.9(1.8)$ & $0.71(-2.4$ to 0.9$)$ & 0.36 \\
\hline $\begin{array}{l}\text { What difference do you think this technique had to your sputum } \\
\text { clearance? } \\
\text { ( } 0 \text { no difference, } 10 \text { most difference) }\end{array}$ & $4.6(3.0)$ & $4.5(2.7)$ & $-0.1(-1.3$ to 1.4$)$ & 0.91 \\
\hline
\end{tabular}

$\mathrm{ACT}$, airway clearance technique; NIV, non-invasive ventilation.

very well, which may have reduced the effect of ACT. However, it is noted that studying more unwell patients would have its own difficulties in terms of the need for treatment changes and ethical considerations.

This study has several limitations. First is the low number of participants, which resulted in early termination of the study. Recruitment, even on a busy CF unit, was difficult due to tight inclusion criteria, especially the need to be stable and being at the end of a hospital admission. Clinical practice and patient preference for early discharge meant that often these patients were sent home to complete their intravenous antibiotics, rendering them unable to complete the study. These difficulties led to a long recruitment time for the study, which was not ideal. Recently O'Neill $e t a l^{21}$ reported similar difficulties with recruitment and participant inclusion in their work looking at the timing of hypertonic saline and ACT, again resulting in early termination of the study. In our clinical practice, NIV is considered to support ACT in patients with $\mathrm{CF}$ with advanced lung disease who are struggling with their usual ACT. In order to replicate this practice in this study, we would have needed to only include patients with a low $\mathrm{FEV}_{1}$; however, due to recruitment, we included all $\mathrm{FEV}_{1}$ ranges, which may explain why differences with NIV were not identified, as patients with good lung function probably do not require NIV assistance to aid them in taking deep breaths. Another limitation, particularly in subjects with higher lung function, was the limited positive pressure support that the Breas iSleep 25 could offer; often these participants were asking for more pressure for a deeper breath which the NIV was unable to provide. We included all ACT techniques to compare against NIV-supported ACT-this decision was taken to ensure familiarity of technique to the patient, but in doing so we accept this introduced more variability in the control arm. It may be that NIV-supported ACT is more effective than some usual ACTs, for example PEP as described by Rodriguez Hortal et al, ${ }^{12}$ but due to the low number of participants it was not possible to analyse the techniques separately.

Although the study was underpowered, we believe it provides important insights into the role of NIV-supported ACT. A recent review of the UK's national data registry has shown that NIV use is increasing with beneficial effects on lung function decline, ${ }^{22}$ and so research investigating the optimal use of NIV for our patients remains essential. From this study we cannot provide definitive conclusions about efficacy, but NIV was well tolerated and improved oxygenation during the intervention. NIV-for select patients-is likely to be where it fits, but further studies are required. With careful clinical consideration, we suggest it should be included in the specialist CF physiotherapist's airway clearance 'toolbox', as concluded by Moran et al. ${ }^{17}$ Low participant numbers and insensitive outcome measures are well-recognised problems of ACT trials. ${ }^{7} 18192123$ Our work provides important context for future ACT trial design as more robust outcome measures need to be developed. Further work needs to be done with these more robust outcome measures to optimise power so that we can better understand who will benefit from this intervention and when it should be introduced.

Acknowledgements Our thanks to Professor Stuart Elborn for his helpful discussions of this work and the physiotherapy team of the Royal Brompton Hospital for their help with this research. Finally we would like to thank the patients who participated in the design and completion of this study.

Contributors GS, HP, PA and DB conceived and designed this research. GS recruited the patients, performed treatment, collected clinical data and performed the treatments. HP led the team who completed the independent observer role. GS, WB and NS analysed the data. GS and NS wrote the paper.

Funding The authors have not declared a specific grant for this research from any funding agency in the public, commercial or not-for-profit sectors. 
Competing interests NS has consulted for Vertex Pharmaceuticals, Chiesi, Roche, Pulmocide, PTC Therapeutics and Gilead.

Patient consent for publication Not required.

Ethics approval Ethical approval was granted by the London - Chelsea Research Ethics Committee (reference number 13/L0/0565).

Provenance and peer review Not commissioned; externally peer reviewed.

Data availability statement The datasets generated during and/or analysed during the current study are available from the corresponding author on reasonable request.

Open access This is an open access article distributed in accordance with the Creative Commons Attribution Non Commercial (CC BY-NC 4.0) license, which permits others to distribute, remix, adapt, build upon this work non-commercially, and license their derivative works on different terms, provided the original work is properly cited, appropriate credit is given, any changes made indicated, and the use is non-commercial. See: http://creativecommons.org/licenses/by-nc/4.0/.

\section{REFERENCES}

1. CF Trust 2017 annual report. Reporting and resources, 2018. Available: https://www.cysticfibrosis.org.uk/the-work-we-do/uk-cfregistry/reporting-and-resources

2. Zach MS. Lung disease in cystic fibrosis--an updated concept. Pediatr Pulmonol 1990;8:188-202.

3. Cantin A. Cystic fibrosis lung inflammation: early, sustained, and severe. Am J Respir Crit Care Med 1995;151:939-41.

4. Konstan MW, Berger M. Current understanding of the inflammatory process in cystic fibrosis: onset and etiology. Pediatr Pulmonol 1997;24:137-42.

5. CF Trust. CF trust Factsheet - physiotherapy treatment in cystic fibrosis: airway clearance techniques. CF trust website, 2011. Available: www.cysticfibrosis.org.uk/media/127518/FS Physiotherapy_Airway_Clearance_Mar_13.pdf

6. Cystic Fibrosis Trust. ACPCF standards of care and good clinical practice for the physiotherapy management of cystic fibrosis. 3rd Edition, 2017.

7. Pryor JA, Tannenbaum E, Scott SF, et al. Beyond postural drainage and percussion: airway clearance in people with cystic fibrosis. $J$ Cyst Fibros 2010;9:187-92.

8. Holland AE, Denehy L, Ntoumenopoulos G. Non-invasive ventilation assists chest physiotherapy in adults with acute exacerbations of cystic fibrosis. Thorax 2003;58:880-4.

9. Placidi G, Cornacchia M, Polese G, et al. Chest physiotherapy with positive airway pressure: a pilot study of short-term effects on sputum clearance in patients with cystic fibrosis and severe airway obstruction. Respir Care 2006;51:1145-53.

10. Fauroux $\mathrm{B}$, Boulé $\mathrm{M}$, Lofaso $\mathrm{F}$, et al. Chest physiotherapy in cystic fibrosis: improved tolerance with nasal pressure support ventilation. Pediatrics 1999;103.

11. Dwyer TJ, Robbins L, Kelly P, et al. Non-invasive ventilation used as an adjunct to airway clearance treatments improves lung function during an acute exacerbation of cystic fibrosis: a randomised trial. $J$ Physiother 2015;61:142-7.

12. Rodriguez Hortal MC, Nygren-Bonnier M, Hjelte L. Non-invasive ventilation as airway clearance technique in cystic fibrosis. Physiother Res Int 2017;22. doi:10.1002/pri.1667. [Epub ahead of print: 29 Feb 2016].

13. Stanford G, Parrott H, Bilton D, et al. Positive pressure--analysing the effect of the addition of non-invasive ventilation (NIV) to home airway clearance techniques (ACT) in adult cystic fibrosis (CF) patients. Physiother Theory Pract 2015;31:270-4.

14. De Boeck Ket al. Cystic fibrosis: terminology and diagnostic algorithms. Thorax 2006;61:627-35.

15. Miller MR, Hankinson J, Brusasco V, et al. Standardisation of spirometry. Eur Respir J 2005;26:319-38.

16. Osman LP, Roughton M, Hodson ME, et al. Short-term comparative study of high frequency chest wall oscillation and European airway clearance techniques in patients with cystic fibrosis. Thorax 2010;65:196-200.

17. Moran F, Bradley JM, Piper AJ, et al. Non-invasive ventilation for cystic fibrosis. Cochrane Database Syst Rev 2017;61.

18. Bradley JM, Moran FM, Elborn JS. Evidence for physical therapies (airway clearance and physical training) in cystic fibrosis: an overview of five Cochrane systematic reviews. Respir Med 2006;100:191-201.

19. Sontag MK, Quittner AL, Modi AC, et al. Lessons learned from a randomized trial of airway secretion clearance techniques in cystic fibrosis. Pediatr Pulmonol 2010;173.

20. Kouroukli E, Hatziagorou E, Parisi M, et al. Short-term effect of chest physiotherapy on lung clearance index among patients with cystic fibrosis. J. Cyst Fibros 2018;17S3:S59-S138.

21. O'Neill K, Moran F, Tunney MM, et al. Timing of hypertonic saline and airway clearance techniques in adults with cystic fibrosis during pulmonary exacerbation: pilot data from a randomised crossover study. BMJ Open Resp Res 2017;4:e000168.

22. Archangelidi O, Carr SB, Simmonds NJ, et al. Non-invasive ventilation and clinical outcomes in cystic fibrosis: findings from the UK CF registry. J Cyst Fibros. In Press 2018. doi:10.1016/j. jcf.2018.11.006. [Epub ahead of print: 28 Nov 2018].

23. Main $\mathrm{E}$. What is the best airway clearance technique in cystic fibrosis? Paediatr Respir Rev 2013;14 Suppl 1:10-12. 